\title{
Ubiquitin Homeostasis Is Critical for Synaptic Development and Function
}

\author{
Ping-Chung Chen, ${ }^{1}$ Bula J. Bhattacharyya, ${ }^{2}$ John Hanna, ${ }^{3}$ Heather Minkel, ${ }^{1}$ Julie A. Wilson, ${ }^{1}$ Daniel Finley, ${ }^{3}$ \\ Richard J. Miller, ${ }^{2}$ and Scott M. Wilson ${ }^{1}$ \\ ${ }^{1}$ Department of Neurobiology, Civitan International Research Center, Evelyn F. McKnight Brain Institute, University of Alabama at Birmingham, \\ Birmingham, Alabama 35294, ${ }^{2}$ Department of Molecular Pharmacology \& Biological Chemistry, Northwestern University, Chicago, Illinois 60611, and \\ ${ }^{3}$ Department of Cell Biology, Harvard Medical School, Boston, Massachusetts 02115
}

The ubiquitin-proteasome system (UPS) controls protein abundance and is essential for many aspects of neuronal function. In $a$ taxia $\left(a x^{J}\right)$ mice, profound neurological and synaptic defects result from a loss-of-function mutation in the proteasome-associated deubiquitinating enzyme Usp14, which is required for recycling ubiquitin from proteasomal substrates. Here, we show that transgenic complementation of $a x^{J}$ mice with neuronally expressed ubiquitin prevents early postnatal lethality, restores muscle mass, and corrects developmental and functional deficits resulting from the loss of Usp14, demonstrating that ubiquitin deficiency is a major cause of the neurological defects observed in the $a x^{J}$ mice. We also show that proteasome components are normally induced during the first 2 weeks of postnatal development, which coincides with dramatic alterations in polyubiquitin chain formation. These data demonstrate a critical role for ubiquitin homeostasis in synaptic development and function, and show that ubiquitin deficiency may contribute to diseases characterized by synaptic dysfunction.

\section{Introduction}

Synapses are highly dynamic, requiring remodeling of their protein composition during development (Yi and Ehlers, 2007). Functional changes in synapses rely on regulated protein synthesis and turnover. Central to controlling protein turnover is ubiquitin, which marks proteins for destruction by the proteasome (Wilkinson et al., 1980). Many neurological disorders display changes in both protein turnover and synaptic function, indicating that therapies targeted to the UPS may be beneficial for treating diseases associated with synaptic dysfunction (Hegde and Upadhya, 2007; Lee et al., 2010).

Proteasomes are the site of regulated protein degradation and are essential for controlling the level of many regulatory proteins. Proteasomal degradation is initiated by the binding, deubiquitination, and unfolding of poly-ubiquitinated proteins (Glickman and Ciechanover, 2002). These activities reside on the $19 \mathrm{~S}$ regulatory particle of the proteasome, and changes in the composition of this particle are hypothesized to alter proteasomal degradation

Received June 9, 2011; revised Sept. 19, 2011; accepted 0ct. 14, 2011.

Author contributions: P.-C.C., B.J.B., R.J.M., and S.M.W. designed research; P.-C.C., B.J.B., H.M., J.A.W., and R.J.M. performed research; J.H. and D.F. contributed unpublished reagents/analytic tools; P.-C.C., H.M., and S.M.W. analyzed data; J.A.W. and S.M.W. wrote the paper.

This research was supported by grants from the National Institutes of Health (NS047533 to S.M.W. and GM65592 to D.F.), the Evelyn F. McKnight Brain Institute, and a March of Dimes Basil O'Conner Award. We thank the Transgenic Animal/Embryonic Stem Cell Resource at UAB for generating the Thy $1-U b$ transgenic mice and NIH Grant P30NS47466 for tissue processing and staining. We also thank Drs. Michael Brenner and Scott Philips for their helpful review of this manuscript.

Correspondence should be addressed to Scott M. Wilson, 1825 University Boulevard, Shelby 914, Birmingham, AL 35294. E-mail: wilson@nrc.uab.edu.

J. Hanna's present address: Department of Pathology, Brigham \& Women's Hospital, 75 Francis Street, Boston, MA 02115.

DOI:10.1523/JNEUROSCI.2922-11.2011

Copyright $\odot 2011$ the authors $\quad 0270-6474 / 11 / 3117505-09 \$ 15.00 / 0$ rates (Lee et al., 2010). Three different deubiquitinating enzymes (DUBs), Poh1/Rpn11, Uch37, and Usp14/Ubp6, associate with the 19S particle, and the ubiquitin hydrolase activity of these DUBs increases after proteasome binding (Borodovsky et al., 2001; Crimmins et al., 2006; Lee et al., 2010). Although both ubiquitin hydrolase-dependent and independent activities have been assigned to proteasomal DUBs, their essential activities are not known.

Ubiquitin levels are determined by the rate of ubiquitin synthesis from $U b b, U b c, U b a 52$, and $U b a 80$ mRNAs and the rate of ubiquitin turnover by lysosomes and proteasomes. Gene disruption studies demonstrate the importance of maintaining ubiquitin levels, as loss of $U b b$ expression leads to neurodegeneration and loss of $U b c$ expression results in embryonic lethality (Ryu et al., 2007, 2008). Although ubiquitin transcription is coupled to cellular stress (Finley et al., 1987; Fornace et al., 1989), the importance of ubiquitin recycling at the proteasome to maintain ubiquitin pools has not been demonstrated in mammals. Our studies indicated that synaptic ubiquitin pools are particularly vulnerable to fluctuations in ubiquitin stability due to their remote location away from the site of ubiquitin synthesis (Chen et al., 2009).

We demonstrated that loss of Usp14 results in a developmental disorder in $a x^{J}$ mice due to a block in maturation of the neuromuscular junction (NMJ) (Chen et al., 2009). In this report, we investigated how loss of Usp14 causes this disorder. While wildtype (wt) mice undergo a rapid increase in ubiquitin expression during the first week of postnatal development, Usp14-deficient $a x^{J}$ mice were unable to maintain high levels of ubiquitin during this period. Loss of ubiquitin in $a x^{J}$ mice coincided with a compensatory increase in ubiquitin mRNA levels, indicating that Usp14 is required for postnatal stability of ubiquitin. Restoration of neuronal ubiquitin by transgenic complementation prevented 
a

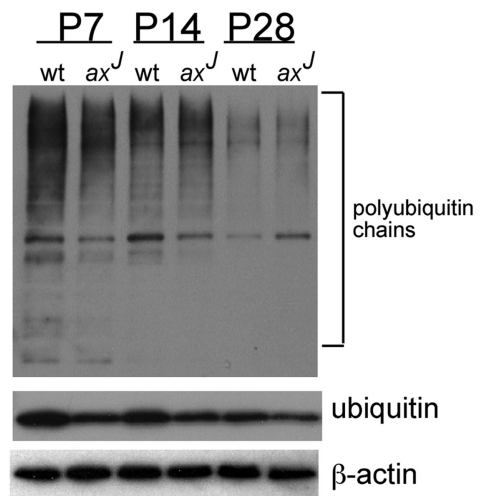

C

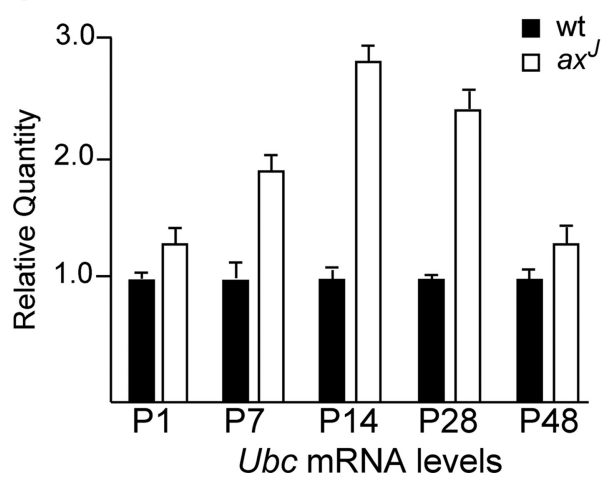

b
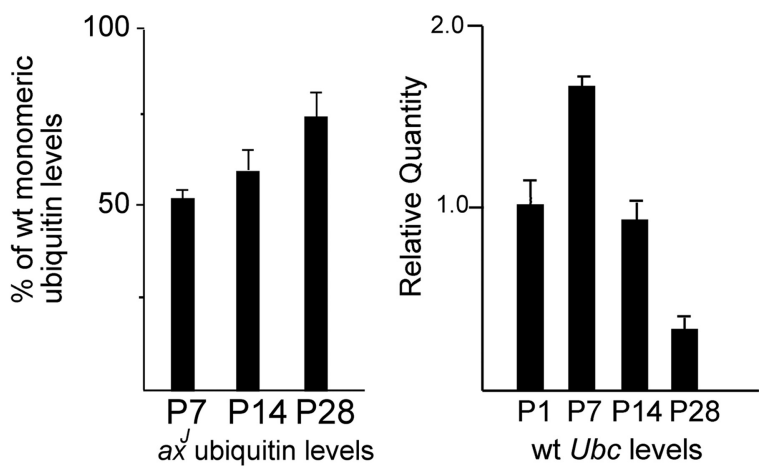

d

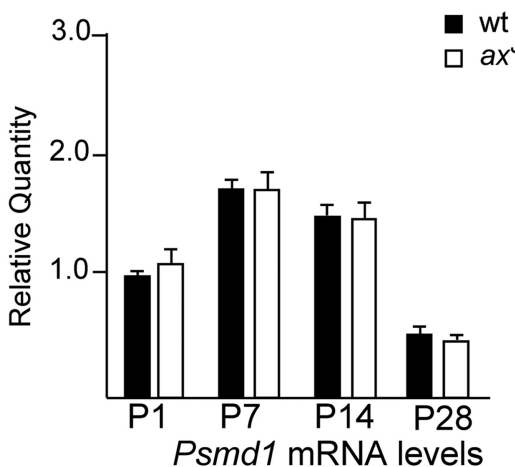

Figure 1. Usp14 is required for maintaining ubiquitin levels during postnatal development. $a$, Representative immunoblot of ubiquitin levels from the brains of P7, P14, and P28 wt and $a x^{\prime}$ mice. $\beta$-Actin was used as a loading control. Graph depicts quantitation of monomeric ubiquitin levels in $a x^{\prime}$ mice relative to wt controls. $\boldsymbol{b}$, Quantitative PCR of $U b c \mathrm{mRNA}$ from brains of wt mice during the first 4 weeks of postnatal development. P1 levels are set at 1.0. $c$, Quantitative PCR of $U b c$ mRNA levels from the brains of wt and $a x^{\prime}$ mice during the first 4 weeks of postnatal development. At each developmental time point, wt levels were set at 1.0. $\boldsymbol{d}$, Quantitative PCR of Psmd 1 levels in wt and ax ${ }^{\prime}$ mice during the first 4 weeks of postnatal development. Wt levels at P1 were set at 1.0. Data are shown as mean $\pm \mathrm{SEM}$, where $n=3$ mice per genotype or time point.

the neuromuscular disease resulting from the lack of Usp14 and restored synaptic transmission at the NMJ, indicating that ubiquitin homeostasis is an essential component of synaptic function. These findings demonstrate a novel role for Usp14 in regulating synaptic ubiquitin pools and indicate that fluctuations in ubiquitin may contribute to other neuronal diseases that exhibit alterations in protein turnover.

\section{Materials and Methods}

Animals. Wild-type C57BL/6J (wt) and Usp14 ${ }^{a x J}$ mice (The Jackson Laboratory) have been maintained in our breeding colony at the University of Alabama at Birmingham, which is fully accredited by the Association for Assessment and Accreditation of Laboratory Animal Care International. Homozygous Usp $14^{a x J}$ mice (which we refer to as $a x^{J}$ mice) were generated by intercrossing $a x \mathrm{~J} /+$ siblings and could be phenotypically identified by 2 weeks of age. Transgenic ubiquitin mice were generated by cloning the last ubiquitin open reading frame of the $U b b$ gene into the Thy1.2 expression cassette, followed by pronuclear injection into C57BL/6J fertilized eggs. Research was conducted without bias toward the sex of animals used for each study. All research complied with the United States Animal Welfare Act and other federal statutes and regulations relating to animals and experiments involving animals, and adhered to principles stated in the Guide for the Care and Use of Laboratory Animals, United States National Research Council.

Immunoblotting. Proteins were resolved on either 8\% Tris-glycine gels or 4-20\% Tris-glycine NUPAGE gels (Invitrogen) and transferred onto PVDF membranes. The Usp14 Ab (Hanna et al., 2006), ubiquitin Mab1510 (Millipore), neurofilament heavy chain Ab (Sigma), Poh1 Ab (Millipore), Uch37 Ab (Abcam), Psmc2 Ab (Santa Cruz), Psmd1 Ab (Santa Cruz), Gapdh Ab (Abcam), anti K48 and K63 ubiquitin Abs (Milli- pore), and anti- $\beta$-tubulin Ab (Developmental Studies Hybridoma Bank) were diluted in PBS containing 3\% BSA. Primary Abs were detected using an anti-mouse or anti-rabbit HRP-conjugated secondary $\mathrm{Ab}$ (Southern Biotechnology Associates) and Luminol reagents (Pierce).

Quantitation of immunoblots. Blots were scanned using a Hewlett Packard Scanjet 3970 and quantitated using UN-SCAN-IT software. Each value represents the average and standard error from four blots using at least three different animals of each genotype.

Labeling of proteasome-associated DUBs. Activity of proteasomal DUBs was conducted as previously described (Crimmins et al., 2006).

Purification of synaptosomal membranes. Membrane fractions were collected as previously described (Chen et al., 2009).

Quantitative PCR. Total RNA was isolated using RNA-STAT60 (TelTest), and $2 \mu \mathrm{g}$ of the RNA was then reverse transcribed using the Applied Biosystems GeneAmp Gold RNA PCR Reagent Kit. Real-time PCRs were set up in triplicate using TaqMan gene assays and amplified in an Applied Biosystems Step-One instrument. $\Delta \Delta$ CCT curves were generated using Poh1 (Mm00451955_m1), Ubc (Mm01201237_ m1), Usp14 (Mm00458097_m1), Uch37 Mm00497950_m1), Psmc2 (Mm00803207_m1), Psmd1 (Mm01295362_m1), Uchl-1 (Mm00495900_ m1), or Uchl-3 (Mm03040818_s1) Taqman gene assays with 18S and Gapdh (Mm03302249_g1) TaqMan gene assays as internal standards. Quantitative PCR results are shown as the standard deviation of three different amplifications from RNA that was reverse transcribed from three different mice. Individual gene assay kits were purchased from Applied Biosystems for each of the RNAs analyzed. Paired $t$ tests were conducted on relative quantity (RQ) values for each group to determine their significance.

Muscle wet weight analysis. Gastrocnemius muscles were collected from 2-, 8-, and 12-week-old wt, $a x^{J}$, and $a x^{J} T g U b$ mice. Muscle weights 
b

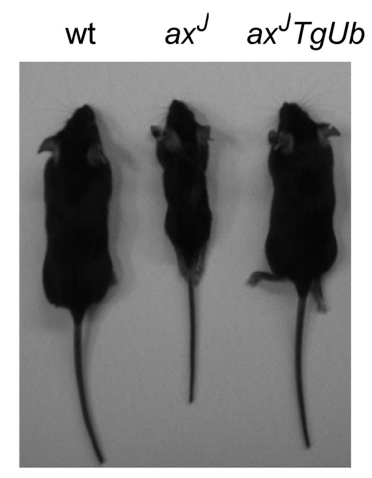

e

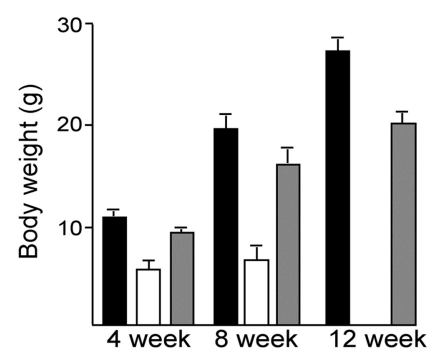

C

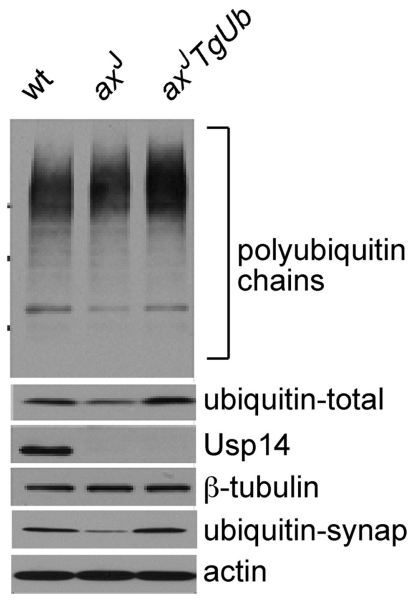

f

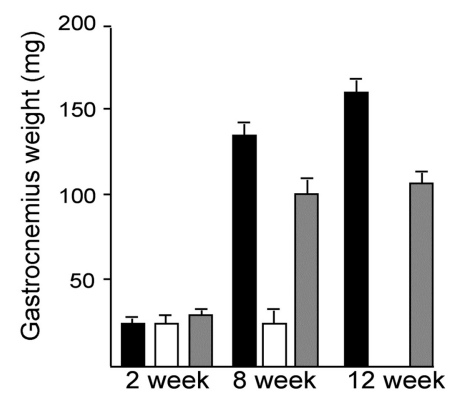

d

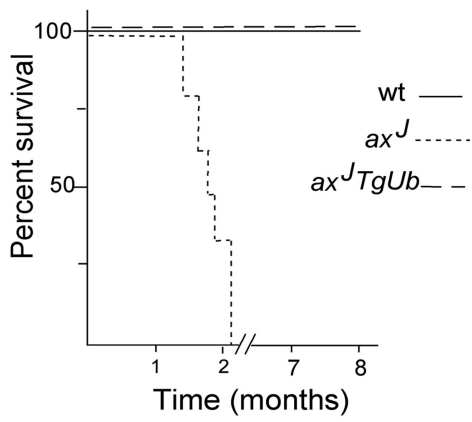

g

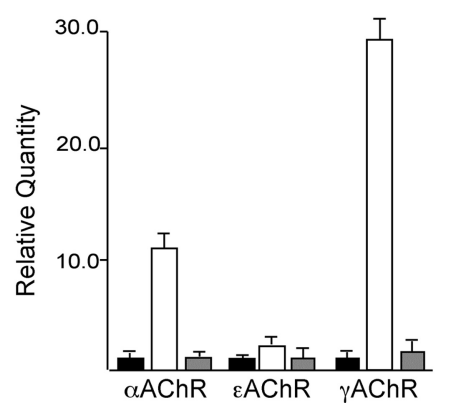

Figure 2. Suppression of the $a x^{\prime}$ neuromuscular disease by transgenic ubiquitin expression. $\boldsymbol{a}$, A single ubiquitin open reading frame of Ubb was cloned behind the Thy 1.2 promoter to drive ubiquitin expression in the nervous system. $\boldsymbol{b}$, Comparison of wt, $a x^{\prime}$, and $a x^{\prime}$ Thy 1 Ubiquitin $\left(a x^{\top} \mathrm{Tg} U b\right)$ mice at 4 weeks of age. c, Representative immunoblot analysis of ubiquitin levels from the brains (total) or synaptosomes (synap) of 4-week-old wt, $a x^{\prime}$, and $a x^{\prime} T g U b$ mice. Usp 14, $\beta$-tubulin, and actin were included as controls. $\boldsymbol{d}$, Eight-month survival curves of wt, $a x^{\prime}$, and $a x^{\prime} T g U b$ mice. $\boldsymbol{e}$, Body weights of wt, $a x^{\prime}$, and $a x^{\top} \operatorname{Tg} U b$ mice ( $n=5$ mice per genotype). $\boldsymbol{f}$, Gastrocnemius muscle wet weights from wt, $a x^{\prime}$, and $a x^{\prime} \mathrm{Tg} U b$ mice ( $n=6$ mice per genotype). $\boldsymbol{g}$, Quantitative PCR of $\alpha, \varepsilon$, and $\gamma$ acetylcholine receptor mRNAs from the tibialis anterior muscles of 4-week-old wt, $a x^{\prime}$, and $a x^{\prime} T g U b$ mice. Wt expression levels of each AChR were set to 1.0. wt (black bars), $a x^{\prime}$ (white bars), and $a x^{\prime} T g U b$ (gray bars). Data are shown as mean \pm SEM, where $n \geq 3$ mice per genotype.

were determined for 6 animals per genotype and values are reported as the average muscle mass \pm SEM.

Rotarod test. Motor coordination and balance were tested using an accelerating rotarod test (ENV-575, Med Associates). The rotarod test was performed by placing a mouse on a motor-driven rotating drum flanked by two larger plates and measuring the time each animal was able to maintain its balance while walking on top of the rod. The speed of the rotarod accelerated from 2.5 to $25 \mathrm{rpm}$ over a $3 \mathrm{~min}$ period. Testing included 3 trials/ $\mathrm{d}$ for $3 \mathrm{~d}$ and the data are presented as an average of the trials ( $n>5$ per genotype).

Elevated beam assay. Mice were analyzed for motor function on a rod with a $2 \mathrm{~cm}$ diameter as previously described (Crimmins et al., 2006). Five animals per genotype were analyzed.

Grip strength test. Mouse grip strength was determined using the San Diego Instrument Grip Strength System. The maximum amount of force produced from both forelimbs and hindlimbs was recorded. Each trial consisted of five repetitions of this assay ( $n>5$ per genotype).

Open field test. Mouse locomotor activity was examined by the Open Field Activity System (ENV-515, Med Associates). Mice were acclimated over a period of $30 \mathrm{~min}$ to the room containing the open field system. Mice were then placed into the open field plastic chamber $(43.2 \times 43.2 \times$ $30.5 \mathrm{~cm}$ ), and their activity was tracked by computer for a period of 5 min. Total distance covered during the 5 min period was recorded for $n>5$ animals per genotype.

NMJ immunostaining and confocal imaging. For whole-mount immunostaining, mice were processed as previously described (Goldman et al.,
1985). Primary Abs and their dilutions were as follows: neurofilament heavy chain, 1:400 (Sigma); neurofilament medium chain, 1:1000 (Sig$\mathrm{ma}$ ); synaptophysin, 1:400 (Millipore); SV2 synaptic vesicle protein, 1:200 (Developmental Studies Hybridoma bank); S100 protein, 1:200 (DAKO); phosphorylated neurofilaments SMI312, 1:400 (Covance). Secondary Abs conjugated with Alexa Fluor 488, 568, or 643 dye (Invitrogen) at a 1:500 dilution for $1 \mathrm{~d}$ at $4^{\circ} \mathrm{C}$. At least $30 \mathrm{NMJs}$ per genotype were analyzed.

Electrophysiology. All experiments were performed in vitro at room temperature on phrenic nerve hemi-diaphragm muscle preparations of 5- to 6-week-old $a x^{J}, a x^{J} T g U b$, and wt littermate control mice. The diaphragm muscles were chosen because these muscles are functional in $a x^{J}$ mice until death (8 weeks). Mice were deeply anesthetized with isoflurane before dissection of the diaphragm muscle. The muscles were perfused with Tyrode's solution ( $137 \mathrm{~mm} \mathrm{NaCl}, 2.8 \mathrm{~mm} \mathrm{KCl}, 1.8 \mathrm{~mm} \mathrm{CaCl}_{2}$, $1.1 \mathrm{~mm} \mathrm{MgCl}_{2}, 11.9 \mathrm{~mm} \mathrm{NaHCO}_{3}, 0.33 \mathrm{~mm} \mathrm{NaH} \mathrm{PO}_{4}, 11.2 \mathrm{~mm} \mathrm{dex}-$ trose, $\mathrm{pH} 7.4$ when bubbled with a mixture of $95 \% \mathrm{O}_{2}$ and $5 \% \mathrm{CO}_{2}$ ) at $30^{\circ} \mathrm{C}$. Intracellular potentials and currents were measured with the Axoclamp2A amplifier system using glass microelectrodes filled with $3 \mathrm{M}$ $\mathrm{KCl}$ of $8-15 \mathrm{M} \Omega$ resistance. Synaptic currents [mini-endplate currents (MEPCs) and EPCs] were obtained at $-70 \mathrm{mV}$ holding potential using a cut diaphragm preparation and a two-microelectrode voltage-clamp system (Hegde and Upadhya, 2007). The currents were digitized at $50 \mathrm{~ms}$ per point and stored, captured, and analyzed using pCLAMP 9 software (Molecular Devices). EPCs were elicited by stimulating the corresponding phrenic nerve with rectangular pulses of $0.05 \mathrm{~ms}$ duration. The fre- 
quencies of MEPCs were captured for $2 \mathrm{~min}$ and quantal content was measured by dividing EPC/MEPC, recorded from the same endplate. Statistical significance was assessed with Student's $t$ test and one-way ANOVA.

\section{Results}

Usp14 is required for stable ubiquitin expression during postnatal development of the nervous system Adult mice lacking Usp14 exhibit reduced free ubiquitin pools and abnormal neuronal activity, indicating that Usp14 is required for maintaining ubiquitin levels in the adult nervous system (Anderson et al., 2005, Lappe-Siefke C et al., 2009, Walters et al., 2008, Wilson et al., 2002). To investigate whether ubiquitin depletion also occurs in Usp 14-deficient $a x^{J}$ mice during postnatal development, we compared ubiquitin levels in the nervous systems of wt and $a x^{J}$ mice during the first 4 weeks of postnatal development (Fig. 1a). In wt mice, the highest levels of ubiquitin protein and mRNA were detected during the first week of postnatal development. In the $a x^{J}$ mice, there was a $50 \%$ reduction in free ubiquitin levels at P7 compared to controls. The levels of free ubiquitin began to rebound in the $a x^{J}$ mice by P14, and were at levels up to $70 \%$ of those observed in control mice by P28. In addition, there was a significant increase in $U b c$ (Fig. 1b,c) mRNA levels in the $a x^{J}$ mice compared to controls, indicating a compensatory response by the $a x^{J}$ mice to try to maintain free ubiquitin pools during this critical time in development. Given the strength of these compensatory responses, the $a x^{J}$ mutation must therefore perturb ubiquitin metabolism to an even greater extent than is apparent from the observed twofold reduction in free ubiquitin protein levels. No differences in the levels of the 19S proteasomal subunit Psmd1 were observed in the wt and $a x^{J}$ mice during this same time period (Fig. 1d), implying that increased ubiquitin gene expression in the $a x^{J}$ mice was not due to a global upregulation of the UPS. Importantly, these developmental changes in ubiquitin expression occurred at the same time points where we have previously identified pathological changes in the central and peripheral nervous systems of the $a x^{J}$ mice (Crimmins et al., 2006; Chen et al., 2009), suggesting that defective proteasome recycling of ubiquitin during the first 2 weeks of postnatal development contributes to the abnormal nervous system development in the $a x^{J}$ mice.

\section{Transgenic expression of ubiquitin prevents early postnatal lethality of Usp14-deficient mice}

Usp14 provides several functions at the proteasome, including ubiquitin-chain trimming, noncatalytic inhibition of the proteasome, maintenance of cellular ubiquitin levels, and regulation of the substrate translocation channel of the proteasome core particle (Anderson et al., 2005; Hanna et al., 2006; Peth et al., 2009; Lee et al., 2010). To determine whether ubiquitin loss is directly responsible for the nervous system defects observed in the $a x^{J}$ mice, we constructed a Thy1.2 ubiquitin transgene (Fig. 2a) to restore neuronal ubiquitin levels in the Usp14-deficient $a x^{J}$ mice. After mating the Thy1.2 ubiquitin transgenic and $a x^{J}$ mice, we analyzed the resultant $a x^{J}$ Thy1.2Ubiquitin $\left(a x^{J} T g U b\right)$ mice for b

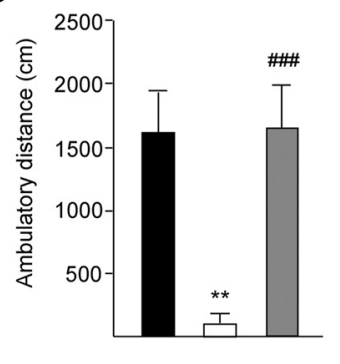

d
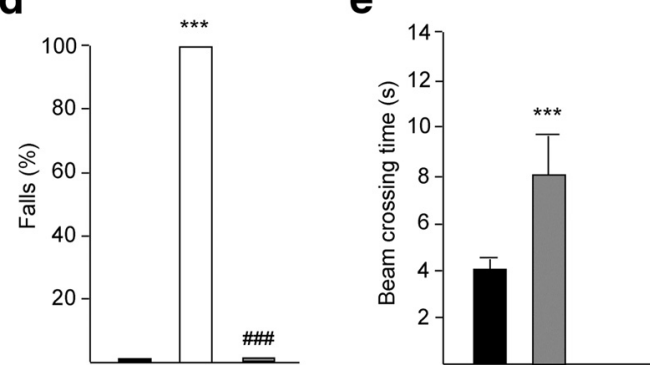

ubiquitin levels, viability, and effects of the ubiquitin transgene on development (Fig. 2b-f). Immunoblot analysis demonstrated that the ubiquitin transgene was able to restore total and synaptosomal ubiquitin levels to the $a x^{J}$ mice (Fig. $2 c$ ). By 8 weeks of age, there was a 2.5-fold increase in body mass and a fivefold increase in muscle wet weights in the $a x^{J} T g U b$ mice compared to $a x^{J}$ mice lacking the ubiquitin transgene (Fig. $2 e, f$ ). While all of the $a x^{J}$ mice died by 8 weeks of age, $a x^{J}$ mice expressing the ubiquitin transgene survived past 8 months of age, with no loss of viability at the endpoint of the experiment (Fig. $2 d$ ).

Increased muscle acetylcholine receptor levels often indicate a reduction in neurotransmission at the NMJ. Our previous studies demonstrated that $a x^{J}$ muscles express high levels of mRNAs encoding the $\alpha^{-}, \varepsilon^{-}$, and $\gamma$-subunits of acetylcholine receptors, which are also observed in denervated rat skeletal muscle (Goldman et al., 1985). The levels of these transcripts returned to wt levels in the $a x^{J}$ mice containing the ubiquitin transgene (Fig. $2 g$ ), indicating that improved neurotransmission at the NMJ may be responsible for the increase in muscle mass in the $a x^{J} \mathrm{TgUb}$ mice.

\section{Restoration of ubiquitin levels corrects structural defects at $a x^{J}$ NMJs}

Loss of Usp 14 expression in $a x^{J}$ mice leads to profound changes in motor function and results in paralysis by $6-8$ weeks of age (Wilson et al., 2002). Increasing ubiquitin expression in the $a x^{J}$ mice significantly improved motor function, in that grip strength, ambulatory distance, and coordination of $a x^{J} \mathrm{Tg} U b$ mice approached levels measured in wt mice (Fig. 3). We then investigated the ability of the ubiquitin transgene to correct the structural and functional defects at the $a x^{J}$ NMJ. In 4-week-old wt mice, the NMJs from the tibialis anterior muscles were well branched and fully innervated (Fig. $4 a, b$ ). In contrast, all $a x^{J}$ motor endplates were defective and contained focal accumulations of neurofilaments, ultra-terminal sprouting, and denervated NMJs. Consistent with the ability of the ubiquitin transgene to improve viability and muscle development, the ubiquitin transgene also improved nerve terminal branching and significantly 
a
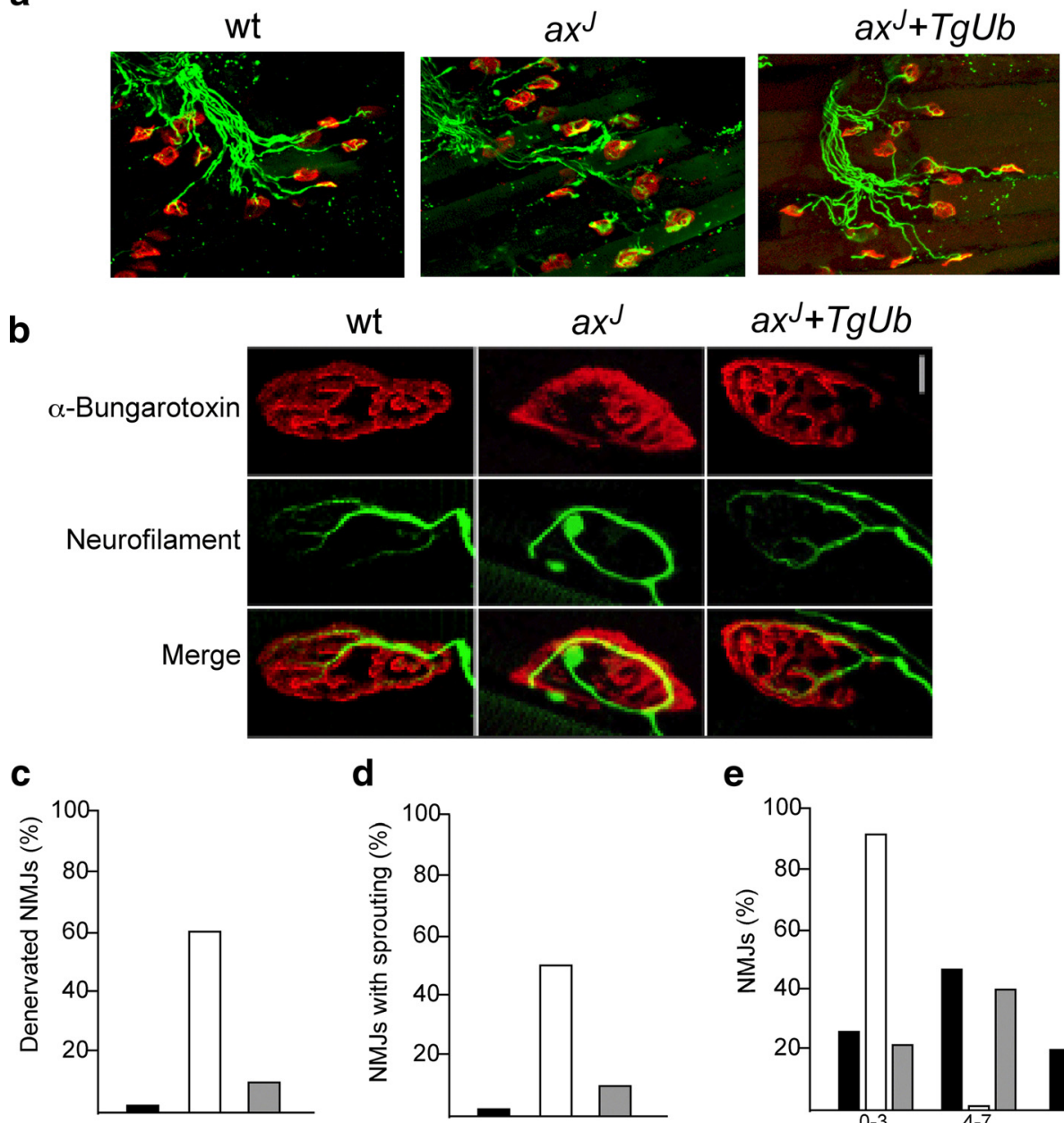

d
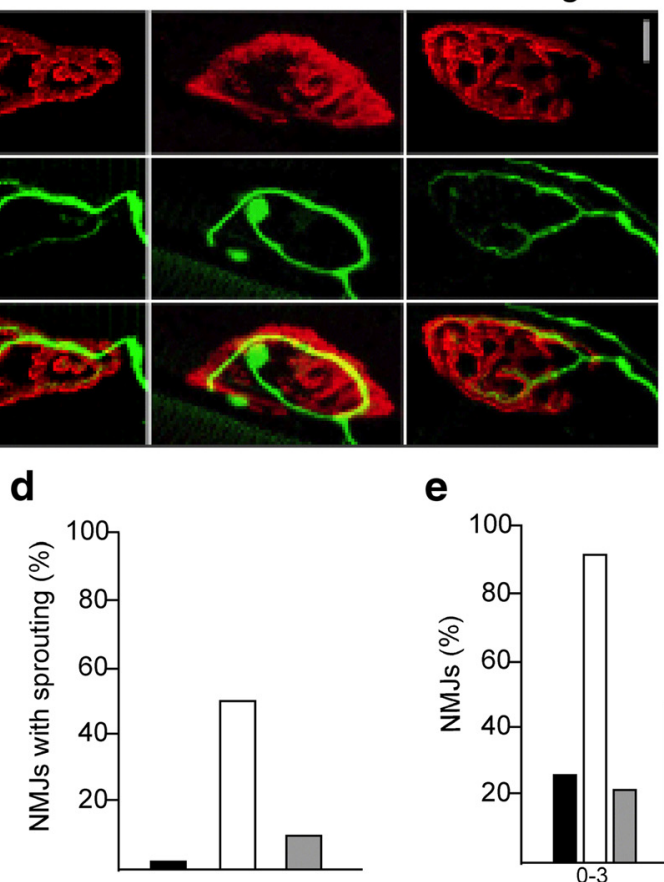

e

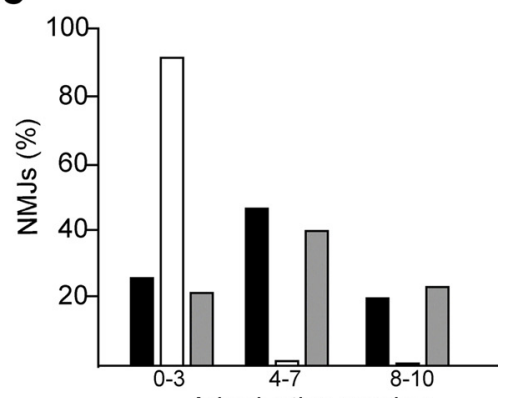

Arborization number

Figure 4. Transgenic expression of ubiquitin corrects the structural and functional deficits at the $a x^{\prime}$ NMJ. $\boldsymbol{a}, \boldsymbol{b}$, Low $(\boldsymbol{a})$ and high $(\boldsymbol{b})$ magnification of whole-mount immunostaining of tibialis anterior muscles from P28 wt, $a x^{\prime}$, and $a x^{\top} \mathrm{Tg} \mathrm{Ub}$ mice. Muscle fibers were stained with antibodies for neurofilaments (green) and acetylcholine receptors were stained with TRITC- $\alpha$-bungarotoxin (red). $\boldsymbol{c} \boldsymbol{e} \boldsymbol{e}$, Quantitation is shown as a percentage change in the numbers of denervated NMJs (c), NMJs containing terminal sprouting (d), and the extent of NMJ arborization (e) in wt (black bars), $a x^{\prime}$ (white bars), and $a x^{J} T g U b$ (gray bars) mice at 4 weeks of age.

corrected the nerve terminal denervation and motor endplate terminal sprouting observed in the $a x^{J}$ mice (Fig. 4).

\section{Ubiquitin is required for maintaining synaptic function}

The $a x^{J}$ mice exhibit defective synaptic transmission in the central and peripheral nervous systems, which include a decreased frequency of spontaneous and quantal release at peripheral synapses and reduced short-term synaptic plasticity at central synapses (Wilson et al., 2002). When we examined the ability of the ubiquitin transgene to correct the synaptic defects of the $a x^{J}$ mice, a twofold increase in spontaneous release, measured as the frequency of MEPCs, was observed in the NMJs from the $a x^{J} \mathrm{TgUb}$ mice compared to the $a x^{J}$ mutant mice (Fig. $5 a, b$ ). This increase in MEPC frequency accompanied a significant reduction in MEPC amplitudes in the $a x^{J} T g U b$ mice, restoring the values to near wt levels (Fig. 5c).

The presence of giant MEPCs is a characteristic feature of the motor endplates from the NMJs of the $a x^{J}$ mice (Wilson et al., 2002). With the ubiquitin transgene, almost all of these giant MEPCs were replaced with lower-amplitude high-frequency MEPCs (Fig. 5a,c). A 14\% increase in evoked release, measured as EPC amplitudes, was also observed in the $a x^{J} T g U b$ mice compared to the $a x^{J}$ mice (Fig. $5 d$ ). These changes resulted in a two- fold increase in quantal release from the NMJs isolated from the $a x^{J}$ TgUb mice (Fig. 5e).

We have previously shown that short-term plasticity, measured as paired-pulse facilitation (PPF), is significantly decreased in the $a x^{J}$ hippocampus when compared to wt controls (Wilson et al., 2002). As in the CNS, there was no measurable PPF at short interpulse intervals (10-20 ms) in the NMJ synapses from the $a x^{J}$ mice (Fig. 5f,g). In contrast, similar to what was observed for the wt endplates, facilitation of paired pulses was evident at short interpulse intervals in the NMJs from the $a x^{J} \mathrm{TgUb}$ mice (Fig. $5 g$ ). These results indicate that the nerve-terminal release of acetylcholine, which was altered in the $a x^{J} \mathrm{NMJs}$, was corrected in the $a x^{J} \mathrm{TgUb}$ mice. The maintenance of ubiquitin pools by Usp14 is therefore essential for the proper structural development of the $\mathrm{NMJ}$ as well as for normal synaptic transmission.

\section{Ubiquitin signaling during postnatal nervous system development}

The importance of the UPS during embryonic development of the nervous system has been demonstrated in both invertebrate and vertebrate animal models (Segref and Hoppe, 2009). To investigate the temporal regulation of the UPS during postnatal development of the nervous system, we examined the expression 
a

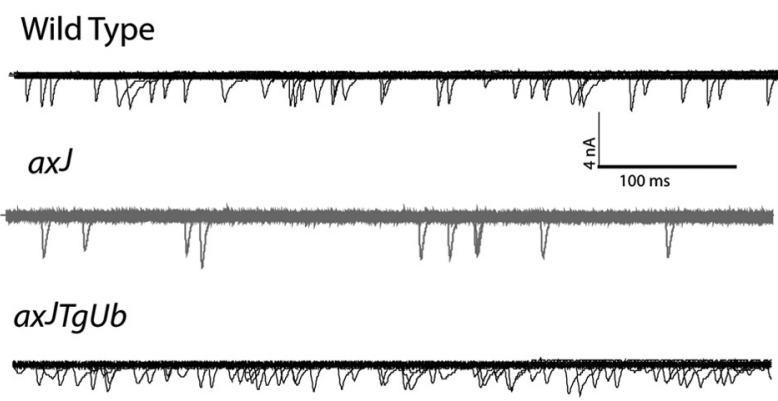

C

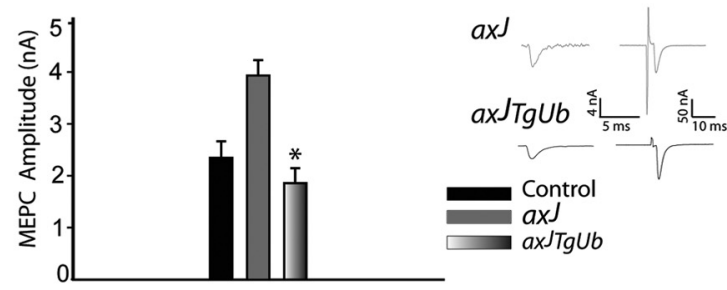

d

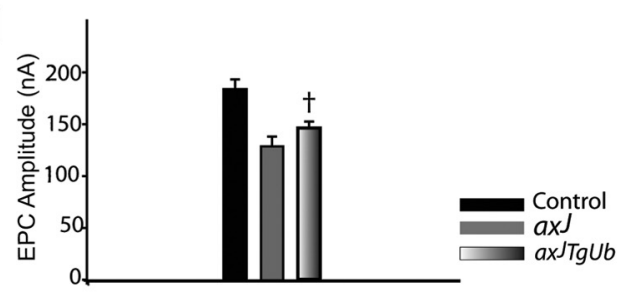

f

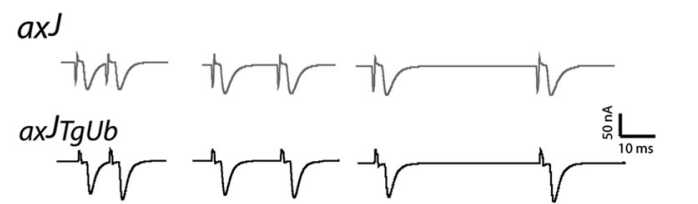

b
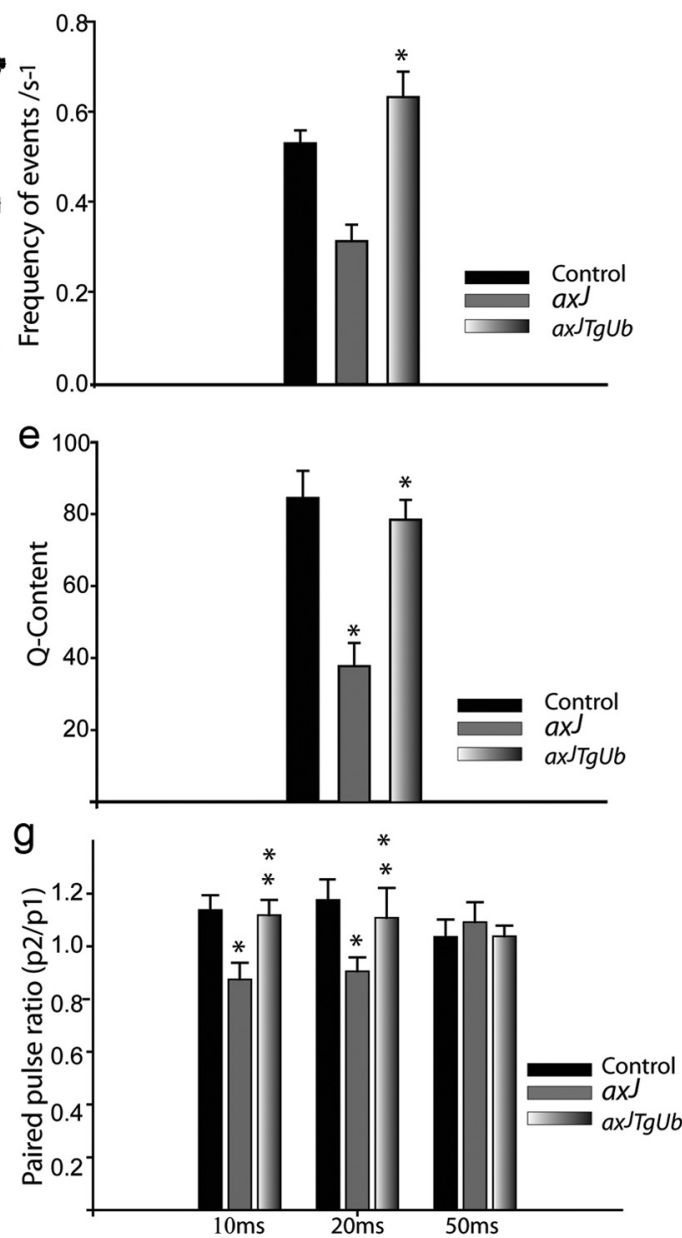

Figure 5. Ubiquitin is required for synaptic transmission at the NMJ. $\boldsymbol{a}$, Representative traces of spontaneous synaptic activity measured as MEPCs (voltage clamped at $-70 \mathrm{mV}$ ) from wt, $a x$ ', and $a x^{\prime} T g U b$ mice. $\boldsymbol{b}$, Transgenic expression of ubiquitin increases MEPC frequency in $a x^{\prime}$ mice. A $106 \%$ increase in MEPC frequency was observed between $a x^{\prime}\left(n=36\right.$ endplates from 8 mice) and $a x^{\prime} T g U b$ mice ( $n=15$ endplates from 4 mice). c, Giant MEPC amplitudes found in $a x^{\prime}$ NMJs ( $n=67$ endplates from 8 mice) were corrected in ax $T g U b$ mice ( $n=19$ endplates from 7 mice). Inset shows representative traces of MEPCs and EPCs captured from $a x^{\prime}$ and $a x^{\prime} \mathrm{TgUb}$ NMJs. $\boldsymbol{d}$, A $14 \%$ increase in EPC amplitudes was observed in the endplates of $a x^{\prime} \mathrm{Tg} U b$ mice ( $n=27 \mathrm{endplates} \mathrm{from} 5$ mice) compared to $a x^{\prime}$ mice ( $n=73$ endplates from 6 mice). e, Quantal content was significantly lower in ax ${ }^{\prime}$ mice ( $n=20$ endplates from 6 mice) than wt littermate controls ( $n=17$ endplates from 6 mice). A $107 \%$ increase in quantal content was observed in the $a x^{J} T g U b$ mice $\left(n=19\right.$ endplates from 4 mice) compared to $a x^{J}$ mice. $f$, PPF was restored in ax $T g U b$ mice. Representative traces of three different interstimulus interval EPCs, ranging from 10 to $50 \mathrm{~ms}$, showing that PPF was increased in ax ${ }^{\prime} \mathrm{T} U \mathrm{Ub}$ mice compared to ax $\mathrm{x}^{\prime}$ mice. $\boldsymbol{g}$, The graph depicts the average paired-pulse ratio (second EPC/first EPC: $\left.p_{2} / p_{1}\right)$ obtained from wt controls ( $n=14$ endplates from 4 mice) and $a x^{\prime}\left(n=18\right.$ endplates from 6 mice) and $a x^{\prime} T g U b$ mice $(n=6$ endplates from 5 mice) at 10, 20, and $50 \mathrm{~ms}$. Data are shown as mean $\pm \mathrm{SEM}$, where $n=6$ mice per genotype. ${ }^{*} p<0.001$ (compared to wt), ${ }^{\dagger} p<0.01$ (compared to $a x^{\prime}$ ), and ${ }^{* *} p<0.05$ (compared to ax').

of proteasomal subunits, ubiquitin hydrolases, and ubiquitin by quantitative PCR and immunoblotting during the first 4 weeks of postnatal development. All proteasomal components examined (Poh1, Usp14, Uch37, Psmc2, and Psmd1) exhibited a similar time course of expression, with the highest mRNA levels occurring at P7 and steadily declining by P28 (Fig. $6 a$ ). Immunoblot analysis of these proteasomal-associated proteins also revealed a robust expression of UPS components at P1-P7, followed by a steady decline by P28 (Fig. 6b). In contrast, UPS components that are not thought to be associated with the proteasome (Uchl-1 and Uchl-3) were not induced at P7.

In addition to its role in protein degradation, ubiquitin also influences many cellular signaling pathways through nonproteolytic mechanisms. These pathways regulate protein kinase activation, DNA repair, vesicle trafficking, and receptor-mediated endocytosis (Chen and Sun, 2009). We used anti-ubiquitin antibodies to differentiate between ubiquitin chains produced by internal lysine 48-linkage, which is used for protein degradation, and those generated by internal lysine 63-linkage, which is fre- quently dedicated to nonproteolytic signaling. Immunoblot analysis of the K48-linked chains in neurons revealed a complex pattern of ubiquitinated protein conjugates that was highest during the first week of postnatal development and then rapidly decreased by P28 (Fig. 6c). In contrast, high-molecular-weight proteins conjugated with K63-linked chains steadily increased to their highest level by postnatal week 4 . These results are suggestive of a high, transient demand on the UPS to reshape the protein composition of synaptic terminals during postnatal development of the nervous system.

Proteasomal deubiquitinating activity during development To assess the enzymatic activity of Usp14 during these same developmental periods, we used a HA-VME-Ub probe that only labels catalytically active, proteasome-bound DUBs, such as Usp14 and Uch37 (Borodovsky et al., 2001; Crimmins et al., 2006). Analysis of wt proteasomal brain extracts demonstrated that the amount of catalytically active proteasomal DUBs reached its highest level by P7 and gradually decreased during the next 2 

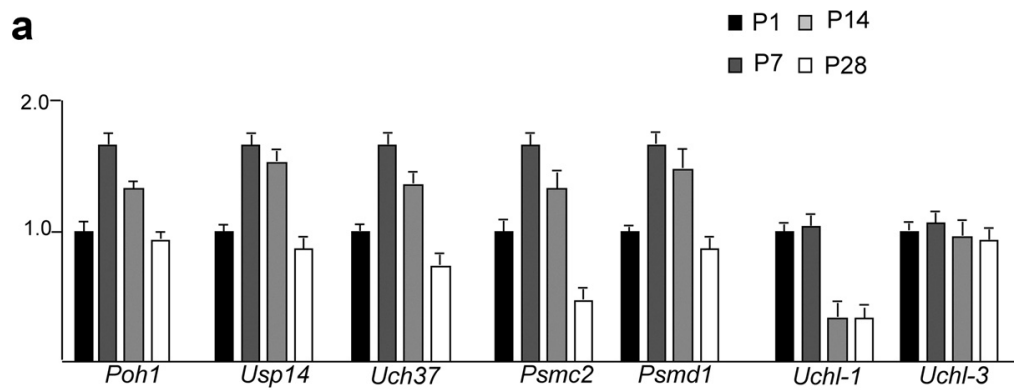

b

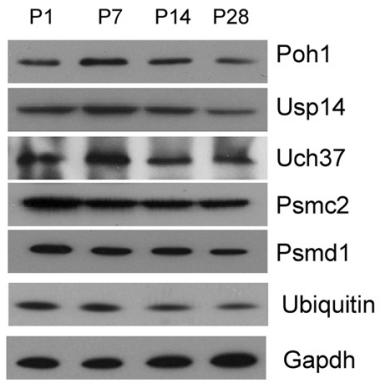

C

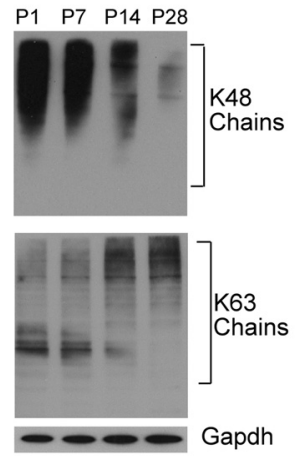

d $\begin{array}{lllll}\text { P1 } & \text { P7 } & \text { P14 } & \text { P21 } & \text { P28 }\end{array}$

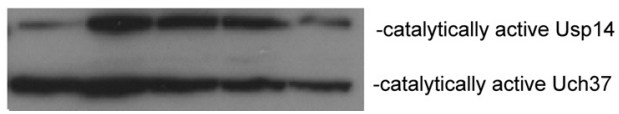

Figure 6. Postnatal expression of UPS components in the nervous system. $\boldsymbol{a}$, Quantitative PCR of proteasome-associated (Poh1, Usp14,Uch37, Psmc2, and Psmd1) and nonassociated (Uchl-1 and Uchl-3) genes in the brains of wt mice during the first 4 weeks of postnatal development. Data are shown as mean \pm SEM, where $n=3$ mice per genotype. P1 values are set to 1.0. $\boldsymbol{b}$, Representative immunoblot of proteasome-associated proteins and ubiquitin from the brains of wt mice during the first 4 weeks of postnatal development. Gapdh was used as a loading control. c, Representative immunoblot of K48- and K63-linked ubiquitin chains from brain extracts of wt mice during development. Gapdh was used as a loading control. $\boldsymbol{d}$, Activity labeling assay of proteasomes from wt mice using a HA-VME-Ub probe, showing the amount of catalytically active Usp14 and Uch37 during the first 4 weeks of postnatal development.

weeks of development (Fig. 6d). Together, these data indicate a critical window for UPS function during the first 2 weeks of neuronal development.

\section{Discussion}

This paper demonstrates the importance of the proteasomeassociated DUB Usp14 in maintaining ubiquitin levels required for proper synaptic development and function. Ubiquitin is essential for the regulated degradation of intracellular proteins by the proteasome, and recent studies have indicated that a balance of ubiquitin conjugation and deconjugation is essential for the ubiquitin-signaling events required for proper synaptic development (DiAntonio et al., 2001, Watts et al., 2003; McCabe et al., 2004). Our findings demonstrate that the maintenance of ubiquitin levels within neurons relies not only on the rapid induction of de novo synthesized ubiquitin but also on the actions of the proteasomal DUBs to prevent inappropriate ubiquitin degradation by the proteasome. Since our data indicate that not all proteasomes contain Usp14 (Crimmins et al., 2006), regulating the interaction of Usp 14 with the proteasome could provide a mechanism for controlling ubiquitin degradation.

Ubiquitin-dependent protein turnover is required for the targeting and formation of synaptic connections during neuronal development. We observed abundant levels of polyubiquitin chains in the nervous system during the first 2 weeks of postnatal development, indicating intense protein turnover during this time period. As the animal enters adulthood, the number of newly formed synapses and the level of polyubiquitin chains are greatly reduced. In the absence of Usp14, ubiquitin turnover by the proteasome during development is greatly enhanced. Due to the increased number of proteins targeted to the proteasome during development, Usp14 may therefore play an essential role in preventing proteasomal degradation of ubiquitin. Consistent with this idea, we observe the peak increase in ubiquitin gene expression at $\mathrm{P} 14$ in the $a x^{J}$ mice, indicating that ubiquitin loss is the greatest during this developmental time point. Even with this dramatic increase in ubiquitin gene expression in $a x^{J}$ brains, the level of monomeric ubiquitin is still only $60 \%$ the level observed in wt brain extracts. Usp14 may therefore be essential for maintaining ubiquitin levels during times of increased proteasome activity such as that seen during nervous system development.

Recent studies indicated that Usp14 can act antagonistic to protein degradation (Lee et al., 2010). In vitro, proteasomes deficient for Usp14 degrade ubiquitinated proteins at a faster rate (Lee et al., 2010). Therefore the accelerated loss of ubiquitin observed in vivo in Usp14deficient mice during development may also reflect an increase in the rate of proteasomal protein degradation in the absence of Usp14.

Our transgenic rescue experiments demonstrate that loss of ubiquitin pools can lead to impaired development of the nervous system. Transgenic restoration of ubiquitin in $a x^{J}$ mice prevents early postnatal lethality, improves muscle mass and NMJ development, and restores synaptic function due to the loss of Usp14. It remains unclear whether the block in NMJ development in the $a x^{J}$ mice is caused by reduced protein degradation that is secondary to the ubiquitin deficiency, or if other ubiquitin signaling pathways, such as vesicular transport, kinase activation, and receptormediated endocytosis, contribute to the motor endplate disease in the $a x^{J}$ mice. However, our finding that K48-linked ubiquitin chains predominate during postnatal development suggests that large demands are made upon ubiquitin pools during development to target proteins for proteasomal destruction.

The finding that the UPS is intimately associated with synaptic function indicates that ubiquitin conjugation is critical for efficient protein turnover at synaptic terminals. E3 ligases have been implicated in the turnover of presynaptic proteins such as RIM1 and synaptophysin (Wheeler et al., 2002; Yao et al., 2007). In addition, genetic studies in invertebrates have demonstrated that the stability of mitogen-activated protein kinases are also regulated by the UPS, and that changes in protein kinase turnover can directly affect synapse development and function (McCabe et al., 2004; Collins et al., 2006). In addition to protein degradation, ubiquitin signaling is also used for protein trafficking. Studies on 
Saccharomyces cerevisiae demonstrated that ubiquitin overexpression restored viability to clathrin heavy chain-deficient yeast, potentially linking ubiquitin signaling to vesicular transport (Nelson and Lemmon, 1993). Future work will focus on determining the ubiquitin signaling pathway that is altered in the $a x^{J}$ mice.

The most striking phenotypic change observed in the $a x^{J}$ mice is at the motor-neuron endplate. The selective developmental defects at the $a x^{J}$ motor endplate are likely due to a combination of the long distances separating the site of ubiquitin synthesis from the synapse and the high demand on the UPS during development of the motor endplate. Ubiquitin is thought to be synthesized in the cell body and transported down axons via slow component b at a rate of $3 \mathrm{~mm} / \mathrm{d}$ (Bizzi et al., 1991). In newborn mice, the sciatic nerve, which innervates the distal leg muscles, approaches $1 \mathrm{~cm}$ in length and would therefore require several days for de novo synthesized ubiquitin to be transported to the motor endplate, making this an inefficient process for regulating ubiquitin levels in distal neuronal compartments. As a result, motor neurons, and other neurons that require ubiquitin to be transported long distances, may be particularly sensitive to local fluctuations in ubiquitin levels.

Previous studies support a developmental regulation of ubiquitin conjugation in the nervous system and indicate that protein degradation by the proteasome is required for normal neuronal development (Flann et al., 1997; Yang et al., 2002). The NMJ undergoes a massive rearrangement in size and structure during postnatal development and transforms from a small "plaquelike" structure to a large "pretzel-like" structure (Sanes and Lichtman, 1999). Changes in components of both the cellular signaling machinery and the structural components of the NMJ are essential for proper postnatal development of the motor endplate, and these changes in protein expression are highly dependent on efficient ubiquitin-dependent proteolysis. By facilitating local recycling of ubiquitin by the proteasome, Usp14 therefore helps control the free ubiquitin levels that are essential for the formation and function of the NMJ.

Many neurological diseases and developmental disorders are associated with alterations in synaptic activity, and our data demonstrate that ubiquitin homeostasis is essential for the proper development and function of synaptic connections. The presence of ubiquitinated protein aggregates in some of these diseases suggests impairment of the UPS. These stable ubiquitinated protein deposits may act to sequester ubiquitin pools and suppress ubiquitin signaling events required for proper synaptic development or function. It will be important to determine whether ubiquitin deficiency contributes to chronic neurological diseases such as Huntington's and Parkinson's, and to determine whether strategies to augment ubiquitin levels might help to improve synaptic function and promote neuronal survival.

\section{References}

Anderson C, Crimmins S, Wilson JA, Korbel GA, Ploegh HL, Wilson SM (2005) Loss of Usp14 results in reduced levels of ubiquitin in ataxia mice. J Neurochem 95:724-731.

Bizzi A, Schaetzle B, Patton A, Gambetti P, Autilio-Gambetti L (1991) Axonal transport of two major components of the ubiquitin system: free ubiquitin and ubiquitin carboxyl-terminal hydrolase PGP 9.5. Brain Res 548:292-299.

Borodovsky A, Kessler BM, Casagrande R, Overkleeft HS, Wilkinson KD, Ploegh HL (2001) A novel active site-directed probe specific for deubiquitylating enzymes reveals proteasome association of USP14. EMBO J 20:5187-5196.

Chen PC, Qin LN, Li XM, Walters BJ, Wilson JA, Mei L, Wilson SM (2009) The proteasome-associated deubiquitinating enzyme Usp14 is essential for the maintenance of synaptic ubiquitin levels and the development of neuromuscular junctions. J Neurosci 29:10909-10919.

Chen ZJ, Sun LJ (2009) Nonproteolytic functions of ubiquitin in cell signaling. Mol Cell 33:275-286.

Collins CA, Wairkar YP, Johnson SL, DiAntonio A (2006) Highwire restrains synaptic growth by attenuating a MAP kinase signal. Neuron 51:57-69.

Crimmins S, Jin Y, Wheeler C, Huffman AK, Chapman C, Dobrunz LE, Levey A, Roth KA, Wilson JA, Wilson SM (2006) Transgenic rescue of ataxia mice with neuronal-specific expression of ubiquitin-specific protease 14 . J Neurosci 26:11423-11431.

DiAntonio A, Haghighi AP, Portman SL, Lee JD, Amaranto AM, Goodman CS (2001) Ubiquitination-dependent mechanisms regulate synaptic growth and function. Nature 412:449-452.

Finley D, Ozkaynak E, Varshavsky A (1987) The yeast polyubiquitin gene is essential for resistance to high temperatures, starvation, and other stresses. Cell 48:1035-1046.

Flann S, Hawkes RB, Riederer BM, Rider CC, Beesley PW (1997) Changes in ubiquitin immunoreactivity in developing rat brain: a putative role for ubiquitin and ubiquitin conjugates in dendrite outgrowth and differentiation. Neuroscience 81:173-187.

Fornace AJ Jr, Alamo I Jr, Hollander MC, Lamoreaux E (1989) Ubiquitin mRNA is a major stress-induced transcript in mammalian cells. Nucleic Acids Res 17:1215-1230.

Glickman MH, Ciechanover A (2002) The ubiquitin-proteasome proteolytic pathway: destruction for the sake of construction. Physiol Rev 82:373-428.

Goldman D, Boulter J, Heinemann S, Patrick J (1985) Muscle denervation increases the levels of two mRNAs coding for the acetylcholine receptor alpha-subunit. J Neurosci 5:2553-2558.

Hanna J, Hathaway NA, Tone Y, Crosas B, Elsasser S, Kirkpatrick DS, Leggett DS, Gygi SP, King RW, Finley D (2006) Deubiquitinating enzyme Ubp6 functions noncatalytically to delay proteasomal degradation. Cell 127:99-111.

Hegde AN, Upadhya SC (2007) The ubiquitin-proteasome pathway in health and disease of the nervous system. Trends Neurosci 30:587-595.

Lappe-Siefke C, Loebrich S, Hevers W, Waidmann OB, Schweizer M, Fehr S, Fritschy JM, Dikic I, Eilers J, Wilson SM, Kneussel M (2009) Ax J mutation of the ubiquitin-specific protease 14 gene alters in vivo $\mathrm{GABA}_{\mathrm{A}}$ receptor $\alpha 1$ subunit surface expression and Purkinje cell function. PLOS Genetics 5:e1000631.

Lee BH, Lee MJ, Park S, Oh DC, Elsasser S, Chen PC, Gartner C, Dimova N, Hanna J, Gygi SP, Wilson SM, King RW, Finley D (2010) Enhancement of proteasome activity by a small-molecule inhibitor of USP14. Nature 467:179-184.

Lee MJ, Lee BH, Hanna J, King RW, Finley D (2011) Trimming of ubiquitin chains by proteasome-associated deubiquitinating enzymes. Mol Cell Proteomics 10:R110.003871.

McCabe BD, Hom S, Aberle H, Fetter RD, Marques G, Haerry TE, Wan H, O'Connor MB, Goodman CS, Haghighi AP (2004) Highwire regulates presynaptic BMP signaling essential for synaptic growth. Neuron 41:891-905.

Nelson KK, Lemmon SK (1993) Suppressors of clathrin deficiency: overexpression of ubiquitin rescues lethal strains of clathrin-deficient Saccharomyces cerevisiae. Mol Cell Biol 13:521-532.

Peth A, Besche HC, Goldberg AL (2009) Ubiquitinated proteins activate the proteasome by binding to Usp14/Ubp6, which causes 20 S gate opening. Mol Cell 36:794-804.

Ryu KY, Maehr R, Gilchrist CA, Long MA, Bouley DM, Mueller B, Ploegh HL, Kopito RR (2007) The mouse polyubiquitin gene UbC is essential for fetal liver development, cell-cycle progression and stress tolerance. EMBO J 26:2693-2706

Ryu KY, Garza JC, Lu XY, Barsh GS, Kopito RR (2008) Hypothalamic neurodegeneration and adult-onset obesity in mice lacking the Ubb polyubiquitin gene. Proc Natl Acad Sci U S A 105:4016-4021.

Sanes JR, Lichtman JW (1999) Development of the vertebrate neuromuscular junction. Annu Rev Neurosci 22:389-442.

Segref A, Hoppe T (2009) Think locally: control of ubiquitin-dependent protein degradation in neurons. EMBO Rep 10:44-50.

Walters BJ, Campbell SL, Chen PC, Taylor AP, Schroeder DG, Dobrunz LE, Artavanis-Tsakonas K, Ploegh HL, Wilson JA, Cox GA, Wilson SM 
(2008) Differential effects of Usp14 and Uch-L1 on the ubiquitinproteasome system and synaptic activity. Mol Cell Neurosci 39:539-548.

Watts RJ, Hoopfer ED, Luo L (2003) Axon pruning during Drosophila metamorphosis: evidence for local degeneration and requirement of the ubiquitin-proteasome system. Neuron 38:871-885.

Wheeler TC, Chin LS, Li Y, Roudabush FL, Li L (2002) Regulation of synaptophysin degradation by mammalian homologues of seven in absentia. J Biol Chem 277:10273-10282.

Wilkinson KD, Urban MK, Haas AL (1980) Ubiquitin is the ATPdependent proteolysis factor I of rabbit reticulocytes. J Biol Chem 255:7529-7532.

Wilson SM, Bhattacharyya B, Rachel RA, Coppola V, Tessarollo L, House- holder DB, Fletcher CF, Miller RJ, Copeland NG, Jenkins NA (2002) Synaptic defects in ataxia mice result from a mutation in Usp14, encoding a ubiquitin-specific protease. Nat Genet 32:420-425.

Yang S, Wang-Su ST, Cai H, Wagner BJ (2002) Changes in three types of ubiquitin mRNA and ubiquitin-protein conjugate levels during lens development. Exp Eye Res 74:595-604.

Yao I, Takagi H, Ageta H, Kahyo T, Sato S, Hatanaka K, Fukuda Y, Chiba T, Morone N, Yuasa S, Inokuchi K, Ohtsuka T, Macgregor GR, Tanaka K, Setou M (2007) SCRAPPER-dependent ubiquitination of active zone protein RIM1 regulates synaptic vesicle release. Cell 130:943-957.

Yi JJ, Ehlers MD (2007) Emerging roles for ubiquitin and protein degradation in neuronal function. Pharmacol Rev 59:14-39. 